\title{
Human factors for the evaluation of the user expertise in the usage of power tools
}

\author{
Sebastian Helmstetter ${ }^{1}$, René Germann ${ }^{1}$, Moez Abbes ${ }^{1}$, Sven Matthiesen ${ }^{1 *}$ \\ ${ }^{1}$ IPEK - Institut of Product Engineering, Karlsruher Institut of Technology (KIT)
}

* Corresponding author:

Prof. Dr.-Ing. Sven Matthiesen

IPEK - Institut of Product Engineering

Karlsruher Institut of Technology (KIT)

Kaiserstraße 10

76131 Karlsruhe

Phone: +49721608-47156

Mail: sven.matthiesen@kit.edu

\begin{abstract}
In order to optimize the use of hand/ power tools, user differences such as the influence of user expertise must be considered in product design. Therefore, product-independent non-applicationspecific variables are needed to objectively capture user expertise. In sports science, the characteristics of movement are used to evaluate the training level. To what extent these variables can be used for product development is unknown. Therefore, a study with 6 male subjects was carried out to analyze correlations between efficiency and characteristics in the movement behavior of the subjects when hammering. Results indicate a clear difference between experienced and less experienced users. Moreover, the movement of the more efficient subjects was spreading less than those of the less efficient ones.
\end{abstract}

\section{Keywords}

User-centered Design, Human-Machine System, Human Factors, User Expertise 


\section{Motivation}

Power tools such as cordless screwdrivers, hammer drills, and angle grinders are used by professional craftsmen, as well as by do-it-yourself workers with lower experience. These two groups differ in their routine and training in the use of power tools, the duration, and frequency of their use of the power tools, and the requirements for working results. Depending on their future use, the user-centered design has the aim to optimize the power tools for a specific user group [1-3]. The experience of a user influences the use of the product, his requirements for the product but also the way he analyzes and evaluates the product [4-6]. User expertise can be divided into novices, specialists, and experts. Most do-it-yourself workers are novices. Professional craftsmen are specialists because they have a complex knowledge about their work, but they are not used to apply this to other problems in general [7]. In the literature, a distinction is often made between novices and experts, so that professional craftsmen can also be regarded as experts.

For the product evaluation of power tools, the behavior of a professional power tool user as well as how he evaluates the usability of a power tool can be taught by tutorials [1]. The training of subjects enables an objective evaluation of power tools by analytically trained subjects who are not specialized in a certain type of power tool. Using this method, the evaluation of new power tools can be made more objective and efficient. However, for a reliable result of this study, it is necessary to monitor the test persons during the tests and detect non-professional behavior for the usage of the power tools. Conventional evaluation criteria for expertise like the available seniority, the degree of education or diploma, and the assumed knowledge [8] are qualified for classical field studies with professional and non-professional subjects, while they cannot be used for testing with trained subjects. They do not have properties like a diploma or seniority that express their expertise. To make sure, that the training is successful, the behavior of the subjects in the use of the power tools has to be analyzed. Existing methods in the state of the research are effortful because they are only valid for a specific application or power tool. Hereby, a lot of effort is needed to collect information about the professional use of the power tool and to evaluate the expertise of subjects by comparing their behavior to professional use [1]. Application unspecific evaluation criteria for expertise that can be used efficiently for every power tool are missing in the state of the research.

Similar to the evaluation of the expert opinion, sports scientists analyze evaluation criteria for the training level of athletes. They focus in particular on the differences in movement between amateur and professional athletes. Hreljac [9] identified differences in the jerk cost of foot movement as an indicator for trained runners and non-runners. Ganzevles, Beek et al. [10] obtained similar results for the analysis of arm movement of elite swimmers and nonprofessional swimmers. There is a higher deviation in acceleration and jerk costs of arm movement for non-professional swimmers compared to acceleration and jerk costs for elite swimmers. A second evaluation criterion for the expertise of a sportsman is the variability in a repeated movement. Significant differences of the variability can be identified e.g. for the novices, experienced and elite performers of Olympic weightlifting [11].

The movement of the hand-arm-system by using a power-tool has similar elements to the movement of the hand-arm-system in sports. Therefore, it can be assumed that similar evaluation criteria in the movement of the hand-arm system can be used to differentiate user expertise as they are used to evaluate training levels in sports science. This thesis leads to the following research question:

Are there measurable characteristics of hand-arm movement when using a hand or power tool that can be used as evaluation criteria for the user's expertise, similar to the jerk cost or variability of movement that has been identified as evaluation criteria for a particular level of training in sports science? 
Therefore, in an experimental study with 6 male subjects that had the task to hammer nails in a wood beam, characteristics in the movement of the hand-arm system and a correlation to their working performance were measured and analyzed.

\section{Materials and Methods}

\subsection{Measurement equipment}

In the experimental study, the movements of the subjects were measured by an optical and an IMU-based motion capture system. As IMU-based motion capture, system the commercial

system Awinda from Xsens was used [12]. Therefore, eleven wireless IMU-tracker were position on the defined spots on the upper body of the subjects. One tacker is located on the back of the right hand. This tracker was used primarily for further evaluation. After calibration, the acceleration of each tracker, its orientation, and the movements of the joints on the upper body were measured. The recording frequency of this system was $60 \mathrm{~Hz}$. The setup of the study and the placement of the measurement technology is shown in Figure 1.

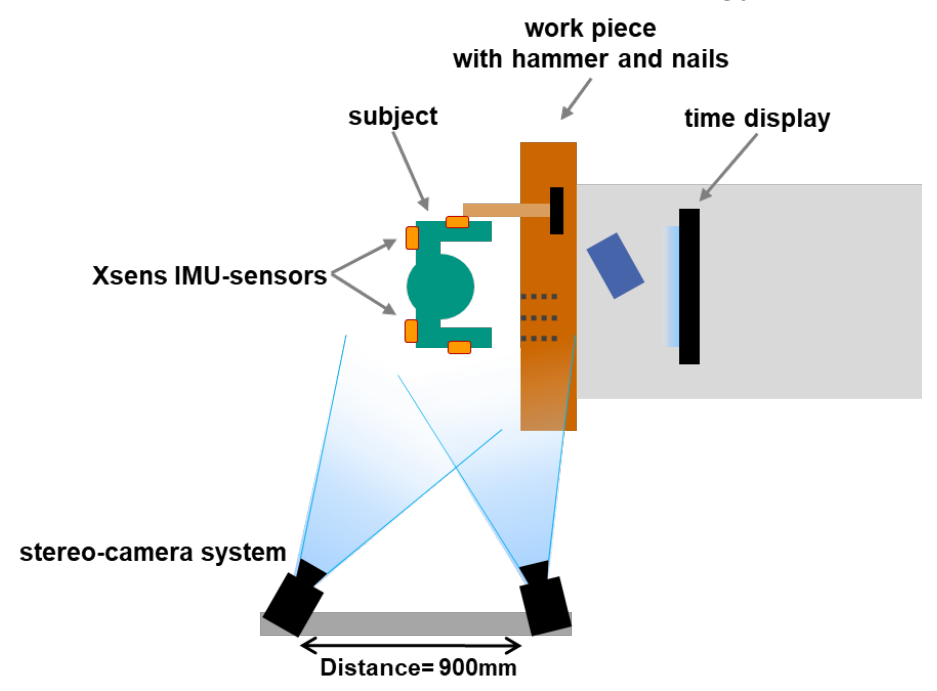

Figure 1: Scheme of the study setup: Subject with IMU sensors from Xsens on the arms and upper body; wood beam for nailing with a high of $100 \mathrm{~cm}$ in front of the subject

The optical motion capture system was based on the pose estimation algorithm OpenPose [13]. Therefore, two synchronized cameras (acA1300-200uc from Basler: recording frequency $=$ up to $200 \mathrm{fps}$, resolution $=1,3 \mathrm{MPix}$, focal length $=8 \mathrm{~mm}$ ) recorded the movements of the subjects from to spots with a distance of $900 \mathrm{~mm}$. In this study, a recording frequency of $60 \mathrm{~Hz}$ and an exposure time of $3,3 \mathrm{~ms}$ was used. To get the movement of the hand-arm-system of the subjects, the OpenPose algorithm (portable demo version v1.6.0) on a windows $p c$ with a NVIDIA Quattro P1000 GPU computed the recorded video files. The results of the algorithm for both cameras - the coordinates of the detected joints in pixel on the video - were computed to 3D-positions by the "stereoCameraCalibration" function of Matlab. Therefore, calibration of both synchronized cameras was conducted with a black and with a grid board with $40 \mathrm{~mm} x$ $40 \mathrm{~mm}$ black squares before the study at a distance of about two meters to the camera.

\subsection{Participants}

During the experimental study, six male subjects have been investigated. All of them were mechanical engineering students or research assistant at the IPEK - institute for product engineering. The subjects aged between 22 and 29 . They had an average height of $187,33 \mathrm{~cm}$ $\pm 8,48 \mathrm{~cm}$. None of the subjects suffered from muscle disorders or other health restrictions. All subjects claimed to be right-handed. Therefore, the camera system was just focused on the 
right hand-arm-system of the subjects. None of the subjects was a professional craftsman or had an education in craft profession. All subjects used power tools regularly for DIY projects (monthly to once a week).

\subsection{Experimental procedure}

At the beginning of each experiment, the subjects were asked about their age, height, righthandedness, and their experience in craftwork. Afterward, the IMU sensors of Xsens ware positioned on the upper body of the subject before the Xsens system was calibrated with a walk over $10 \mathrm{~m}$ (Xsens calibration setting: Npose). After the preparation of the subject, he got the task for the study. The subjects had to hammer as many nails (diameter $=3,4 \mathrm{~mm}$, length $=90 \mathrm{~mm})$ as possible within two minutes with a mechanist hammer $(500 \mathrm{~g}$ head) in a beam $(100 \mathrm{~cm} \times 32 \mathrm{~cm} \times 12 \mathrm{~cm})$ of construction wood (spruce and fir). The beam was placed at a height of $100 \mathrm{~cm}$. The subject stood upright in front of the beam and had any number of nails in a box ahead to perform the experiment. The time was displayed on a monitor in front of the subject. No further instructions were given for body posture, holding the hammer, or carrying out the hammer impacts. The current hammering process could be aborted and started with the next nail when the nail got a bad back. After the time was over, the subject had to stop hammering by themself and the recording of his movement stopped.

\subsection{Evaluation methods}

The study aimed to identify correlations between the hand-arm movement and the efficiency of the behavior of each subject. To rate the efficiency, the number of nails that were hammered within two minutes was compared in the assumption that more professional behavior results in faster work and therefore a higher number of hammered nails. Only nails that were hammered in more than $75 \%$ were counted. Furthermore, the number of impacts per nail, the number of crookedly hammered nails, the hand position on the shaft, and the type of movement were evaluated visually by analyzing the video data in a post-study phase. The hand position on the hammer was classified by the distance between the hammer's head from the gripping position into close to the head, in the middle of the handle, or at the end of the handle. The type of hand-arm movement was classified according to whether the wrist was loose or stiff.

In this paper, only the measurement data of the acceleration of the IMU sensor on the right hand and the joint angle of the right wrist were used for the evaluation of the hand-arm movement. They are both outputs of the Xsens motion capture system. Equivalent to the evaluation in the sports science, the jerk of the hand movement was computed by differentiating the acceleration measured by the IMU sensor on the right hand. The IMU sensor is placed on the back of the right hand. So, the y-axis of the local coordinate system on the sensor points vertically to the ulna from the thumb to the little finger (Figure 2). The joint angle of the wirst is described the clockwise rotation around the $y^{*}$-axis of the second local coordination system with the origin in the wrist. For the evaluation of the subjects by measurement data, the joint angle and acceleration for each impact are calculated. For a general assessment of the evaluation method, sections of the hammer sequences for each subject are superimposed on each other and the reproducibility and characteristics of the movement are assessed. Besides, the visually best subject is compared with the worst by comparing the scatter of the hammer movement between the two subjects. After comparing the hand-arm movement for two seconds of hammering between all six subjects, the variability of the hand-arm movement by every impact is compared between the first subject, who worked the most efficient, and the fifth subject who hammered the least nails. 


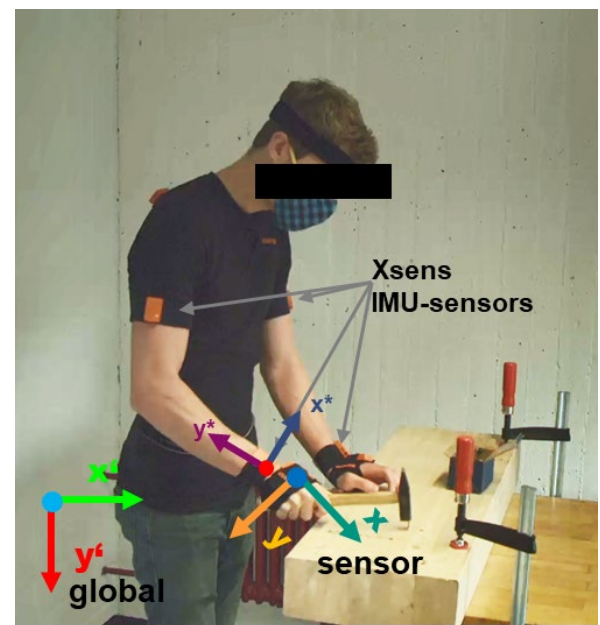

Figure 2: Alignment of the IMU sensor on the back of the hand in the global coordinate system of Xsens

\section{Results}

\subsection{Visual evaluation of the efficiency and user behavior}

The following table is showing the results of the visual evaluation of the efficiency and the way the subjects use the hammer for all of the six subjects:

Table 1: Visual evaluation of the efficiency and the user behavior separated by subjects

\begin{tabular}{|l|c|c|c|c|c|c|}
\hline & subject 1 & subject 2 & subject 3 & subject 4 & subject 5 & subject 6 \\
\hline $\begin{array}{l}\text { number of nails } \\
\text { in 2min }\end{array}$ & 10 & 9 & 10 & 9 & 4 & 8 \\
\hline $\begin{array}{l}\text { number of hits } \\
\text { per nail }\end{array}$ & $\sim 25$ & $\sim 28$ & $\sim 23$ & $\sim 24$ & $\sim 38$ & $\sim 34$ \\
\hline $\begin{array}{l}\text { crookedly } \\
\text { hammered nails }\end{array}$ & - & - & - & - & 1 & 1 \\
\hline $\begin{array}{l}\text { positon of } \\
\text { the hand }\end{array}$ & $\begin{array}{c}\text { end of the } \\
\text { handle }\end{array}$ & $\begin{array}{c}\text { end of the } \\
\text { handle }\end{array}$ & $\begin{array}{c}\text { end of the } \\
\text { handle }\end{array}$ & $\begin{array}{c}\text { end of the } \\
\text { handle }\end{array}$ & $\begin{array}{c}\text { end of the } \\
\text { handle }\end{array}$ & $\begin{array}{c}\text { end of the } \\
\text { handle }\end{array}$ \\
\hline $\begin{array}{l}\text { type of hand- } \\
\text { arm movement }\end{array}$ & loose wrist & loose wrist & loose wrist & stiff wrist & stiff wrist & stiff wrist \\
\hline
\end{tabular}

Subject 5 hammered in significantly fewer nails than the others did in the two minutes with only 4 nails. While the number of impacts per nail at subjects $1-4$ is comparable, it is strongly increased at 5 and 6 . The combination with the higher number of nails hammered in by subject 6 indicates that he made relatively weak, but fast impacts. The difference in the efficiency leads to the hypothesis that the behavior of subject 5 is less professional than the other subjects. This is also indicated by several qualitative characteristics. Subject 5 and 6 are the only ones who drive in nails crooked. The grip position of all subjects was at the end of the shaft. However, subjects 1 to 3 use a loose wrist, while subjects 4 to 6 have a stiff wrist. A loose wrist might allow impacts that are more powerful. This is another indicator for an increased professional usage by the subjects 1 to 3 .

\subsection{Results of the impact characteristic}

The following plots are showing the different characteristics of impacts for each subject. Measured data of a sequence of two seconds during the third nail of every subject. The sequence begins after the subject stops to hold the nail with the left hand. The acceleration is measured at the IMU sensor on the back of the right hand. The joint angel at the right wrist is the output of the Xsens modell. 

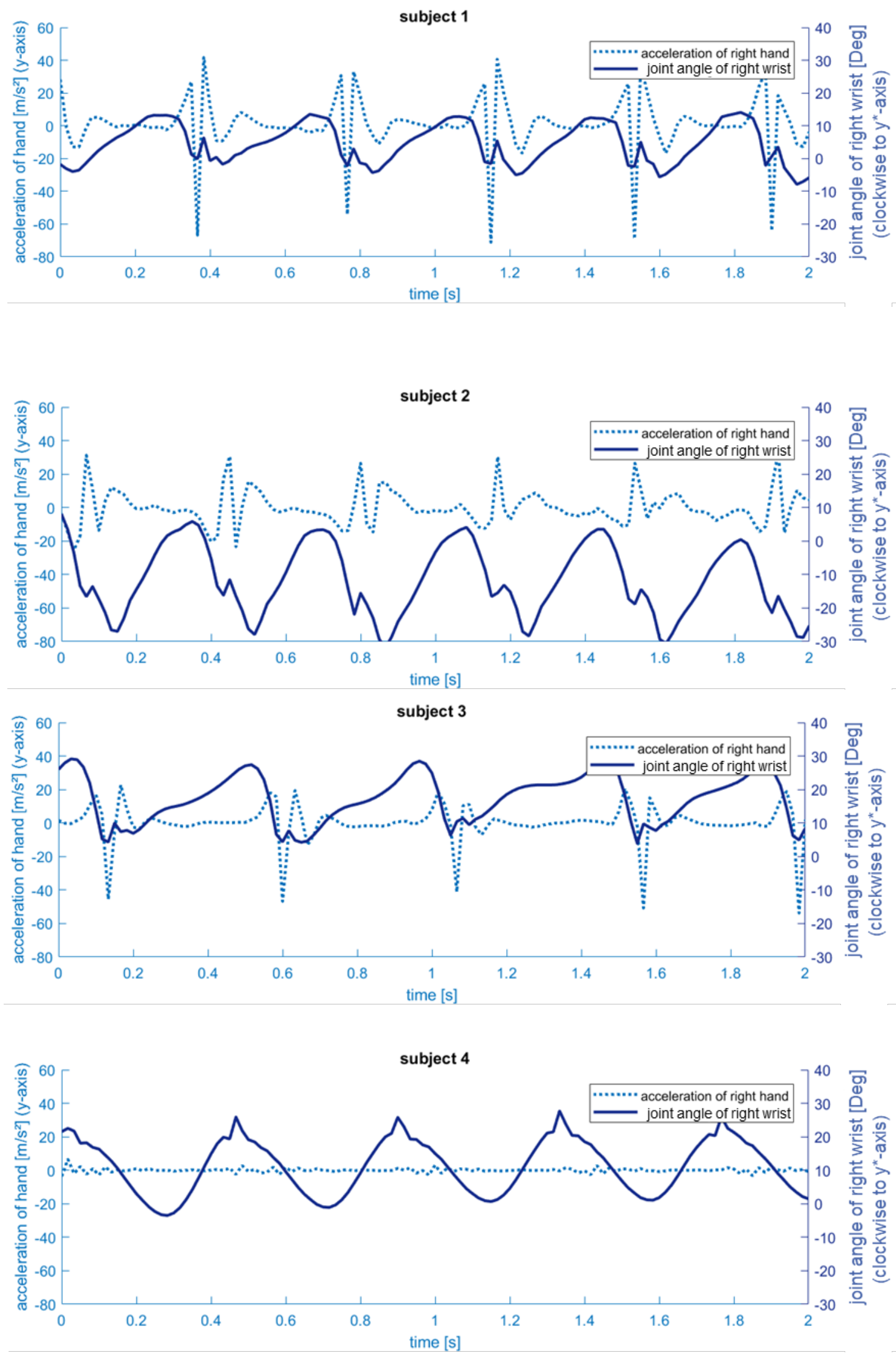

$-26$ 

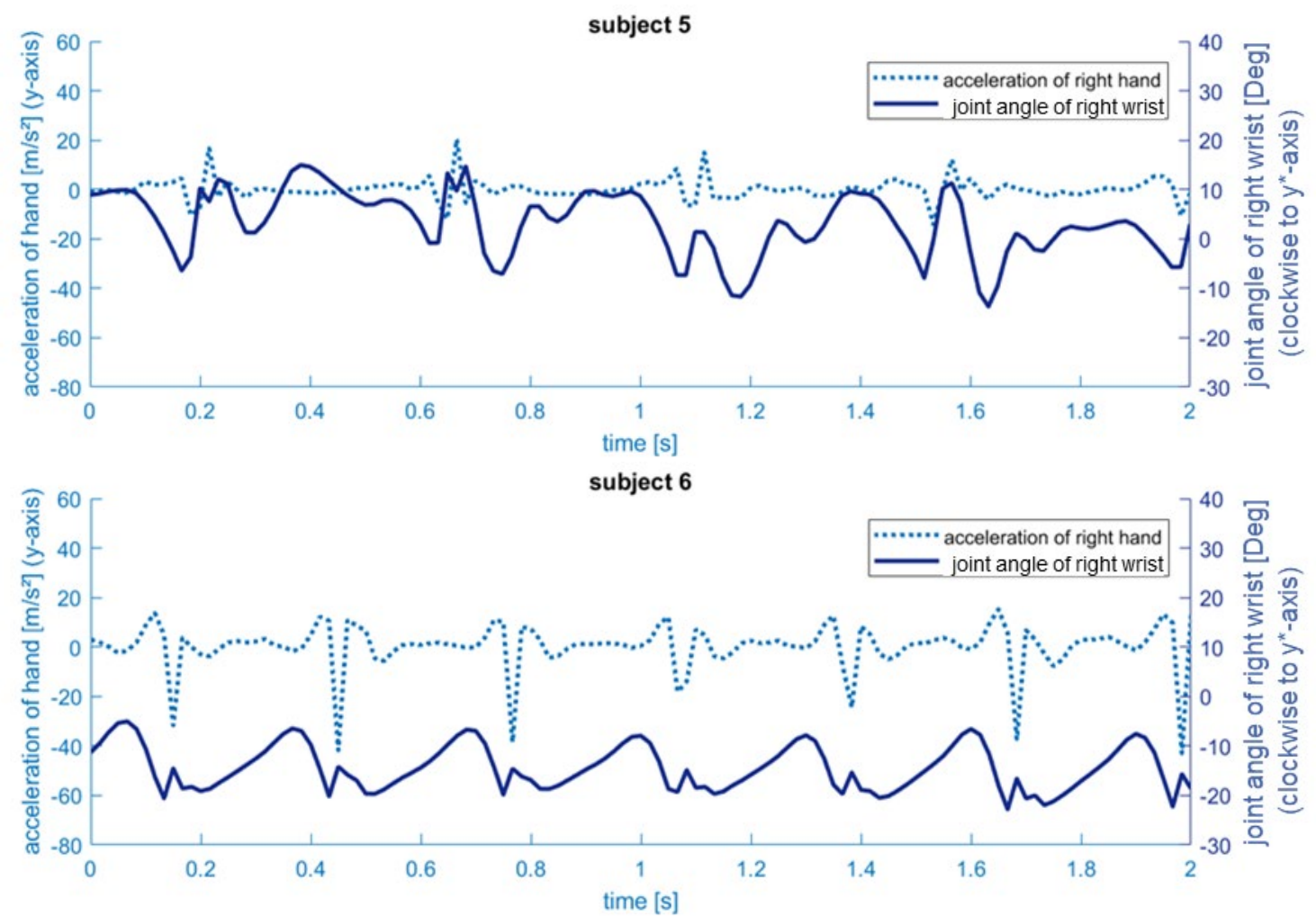

Figure 3: A comparison of the flexion and acceleration data during the experiment on the subject's hand

Figure 3 shows almost sinusoidal curves of hand flexion for the subjects 1 to 4 . In subject 5 , this movement is not visible and in subject 6 , some peeks interrupt the smooth movement when the hand is lowered. The acceleration data of subjects 4 and 5 show a less oscillation at each impact. In subjects 1 to 3 , the impacts can be seen as individual peaks in the acceleration data. The level of maximum acceleration varies widely between subjects. All subjects use an flexation of the wrist of about $25^{\circ}$ to $30^{\circ}$ for the backswing movement.

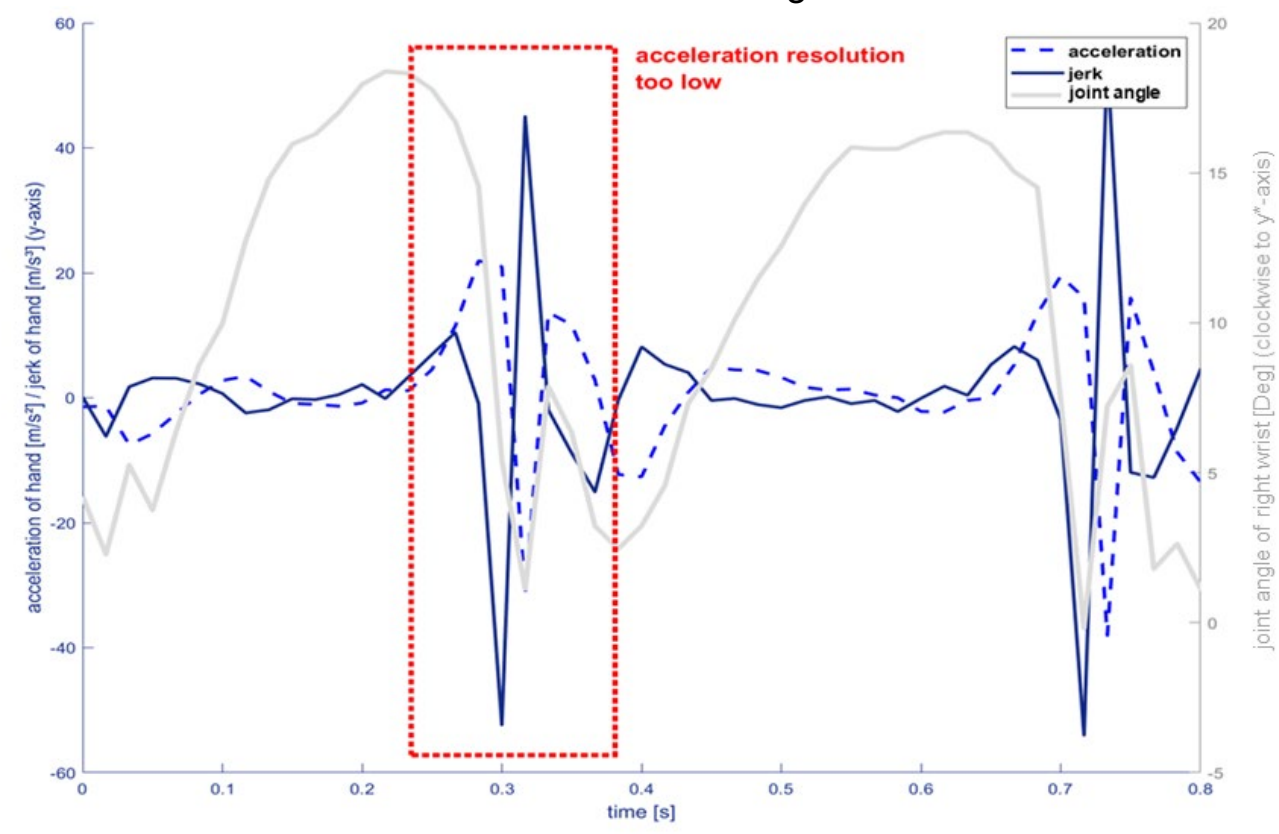

Figure 4: Movement of the right wrist and acceleration and jerk of the IMU sensor on the back of the right hand for two impacts of subject 1 
For the analysis of the jerk, the acceleration measured at the IMU sensor was differentiated. Figure 4 shows the flexation of the wrist, the acceleration of the sensor on the back of the hand, and the jerk measured on the sensor, using the example of two impacts of subject 1 . The data of Figure 4 shows that the acceleration increases too quickly for a continuous course of the accelerations to be recorded at a sampling rate of $60 \mathrm{~Hz}$, especially when the hand is accelerated shortly before the impact. This problem is also transferred to the course of the jerk. Therefore, an evaluation of the jerk and the jerk cost with these data deviates strongly from reality.
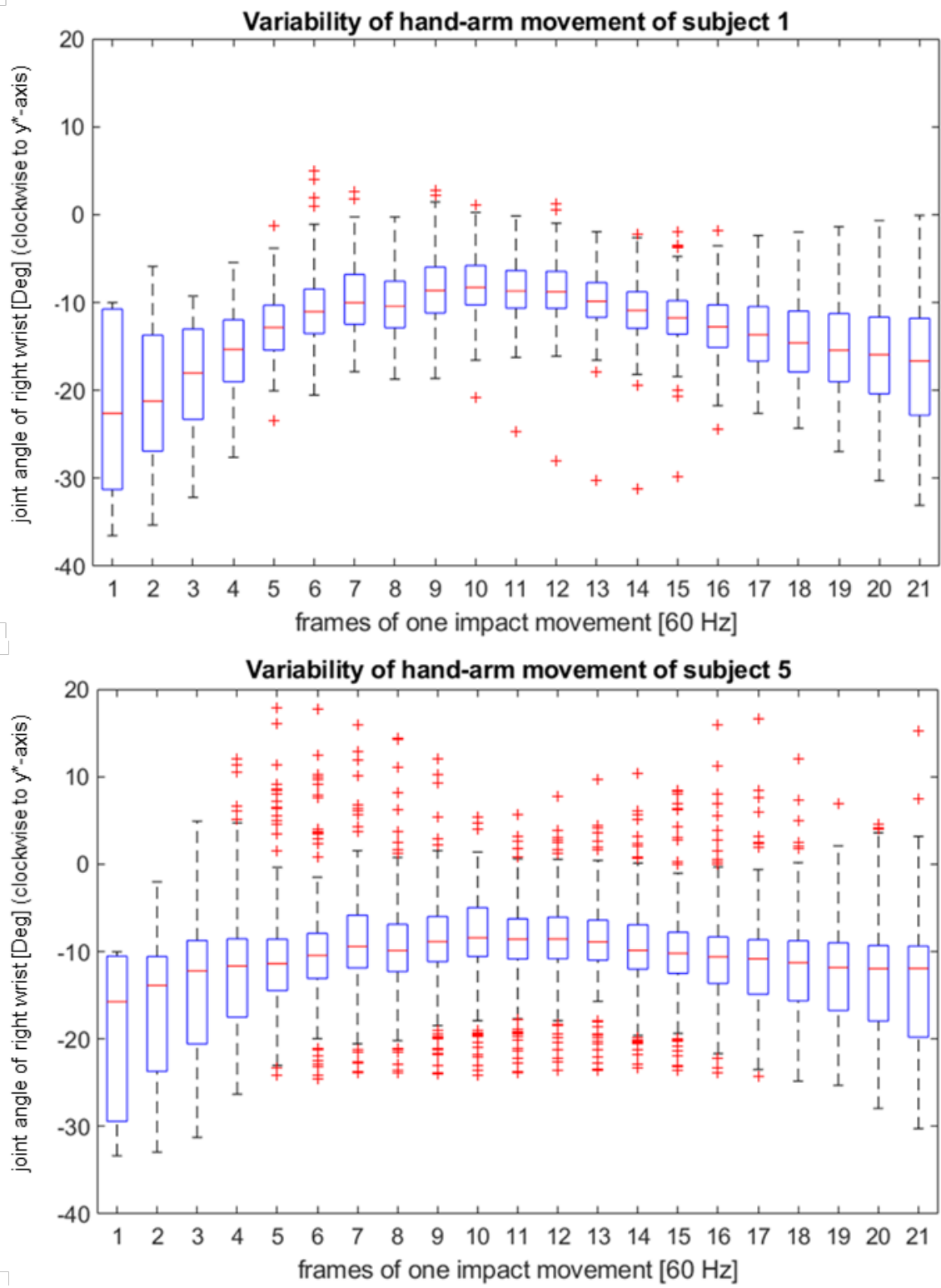

Figure 5: Variability of hand-arm movement evaluated for all impacts of one subject (subject 1: 237 impacts, subject 5: 125 impacts)

Detecting every impact of the hammer on the nail as local minima of the wrist flexation, the backswing movement is separated for every impact. Afterward, the flexation while the backswing movements of one subject are compared to each other. Figure 5 shows the spread of the flexation of the right wrist for subject 1 and subject 5 . The spread of the wrist flexation is displayed as boxplots with whiskers of $95 \%$ for every recoded frame of the flexation (recording frequency: $60 \mathrm{~Hz}$ ). This comparison of subject 1 and 5 shows a significantly greater spread of the flexations from subject 5 to subject 1 . 


\section{Discussion}

Knowing the expertise of subjects is an important prerequisite for the objective and reliable evaluation of the suitability of usage of power and hand tools, an experimental study that analyzes the characteristic of the hand-arm movement for hammering is being conducted. In the study, application-independent evaluation criteria are searched that are suitable for a continuous and automated classification of user expertise. It is already known from sports science that e.g. the jerk during the execution of a repetitive movement can be used as an evaluation criterion to classify different training levels.

To find similar evaluation criteria for the user expertise in the usage of power and hand tools, the results of the study are analyzed to a correlation between the user performance and their hand-arm movement. A significant difference between subjects 1 to 3 and 4 to 6 is the stiffening of the wrist during hammering. This effect can indicate either a higher or less professional behavior. However, the difference in arm posture can be clearly distinguished from the individual swings in subjects 1 to 3 by the less oscillation of the acceleration values at an impact by subjects 4 and 5 , as shown in Figure 3 . Furthermore, subject 6 stands out from the other subjects both in the visual evaluation and in the analysis of the measured data due to the higher impact frequency. These correlations between a characteristic behavior of one of the subjects and the measurement data of the hand-arm movement are potential application specific evaluation criteria for the expertise of the user. They might not be valid for power tools in general but it allows to support and to automate the expertise evaluation by the method of Germann et al. [1].

The variability of the hand-arm movement has already been identified in sports science as an evaluation criterion for different training levels [11]. An extreme value comparison was therefore carried out, comparing the most efficient with the least efficient subject. Variability of the hand-arm movement that is shown by the variability of the joint angele of the right wrist differs significantly between the subject 1 and subject 5 , as it can be seen in Figure 4 . In contrast to the previous criteria, the variability is no evaluation criterion that is assigned to a specific effect of hammering. Based on the finding that this evaluation criterion known from sports science could be identified in the use of hand and power tools, the variability of a repetitive movement seems to be suitable as an application-unspecific criterion for the automated evaluation of user expertise. Thus, the study results indicate that at least one of the evaluation criteria known from sports science is suitable for the evaluation of user expertise in the use of hand and power tools.

One limitation of this study is that no statements can be made about evaluation criteria for the actual differences in hammering between professional craftsmen and nonprofessionals, as no real professional craftsmen participated in the study. Another limitation becomes visible when evaluating the acceleration data for the individual impacts. Even though a recording frequency of $60 \mathrm{~Hz}$ is often used in sports science, the use of hand and power tools can cause high accelerations, which require a higher recording frequency for a reliable evaluation. This is clearly shown in the last phase of acceleration before the hammer impacts the nail. As the resolution is quite low, the evaluation of the jerk would have led to incorrect results in this area. Therefore, the evaluation of the jerk was omitted in the present study. A further study with a higher resolution of the measured data is planned. However, this study cannot be carried out with the motion capture system Xsens, as the recording frequency is determined to $60 \mathrm{~Hz}$. The optical motion capture system, which is based on the pose estimation algorithm OpenPose capture images with a frequency up to $200 \mathrm{~Hz}$ offers a clear advantage. The third limitation of that method is the fact that the evaluation of the spread of repeated movement is only possible for dynamic applications with many repetitions of similar movements. For static applications like the usage of a hammer drill, other measurement methods are needed to evaluate the user expertise. 


\section{Conclusion}

For an objective evaluation of the suitability of usage of hand or power tools, it is necessary to consider the expertise of the test subjects. In the state of research method for a visual evaluation of the expertise of the specific subject behavior during the testing of power tools are shown. To reduce the effort and to objectify these methods, this paper analyzes characteristics in the hand-arm movement of users that can be used for an automated and application unspecific evaluation of the user expertise. Therefore, known measurement characteristics from sports science like the jerk cost and variability of joint angle of the right wrist are used to classify the hand-arm movement of different tool users during the fastening of nails with a hammer.

The main result of the study is that we identified the variability of the hand-arm movement as a potential application unspecific evaluation criterion for the user's performance for hammering. The variability of subject 5 that hammered the fewest nails (4 nails) was significantly higher than the variability of subject 1, who hammered 10 nails.

Furthermore, two potential application-specific evaluation criteria for the user expertise are identified. By analyzing the acceleration of the user's hand, an evaluation for the stiffness of the user's wrist is figured out. The stiffness of the wrist seems like an indicator for the user expertise. The second potential evaluation criterion of user expertise might be the frequency of the hammer impacts. It also can be evaluated by the measurement data of the hand-arm movement. Further studies will focus on using the evaluation criteria to analyze the user behavior in a major study with professional craftsmen and DIY workers to validate these potential criteria for the classification of novice and experts in hammering.

\section{References}

[1] Germann, René ; Jahnke, Björn ; Matthiesen, Sven: Objective usability evaluation of drywall screwdriver under consideration of the user experience. In: Applied ergonomics 75 (2019), S. 170-177

[2] Sauer, Jürgen ; Seibel, Katrin ; Rüttinger, Bruno: The influence of user expertise and prototype fidelity in usability tests. In: Applied ergonomics 41 (2010), Nr. 1, S. 130-140

[3] Matthiesen, Sven; Germann, René: Ansatz zur objektiven und effizienten Erfassung der empfundenen Anwendungseignung von Power-Tool. In: Binz, Hansgeorg; Bertsche, Bernd; Bauer, Wilhelm; Spath, Dieter; Roth, Daniel (Hrsg.): Stuttgarter Symposium für Produktentwicklung SSP 2017: Stuttgart, 29. Juni 2017, Wissenschaftliche Konferenz : Stuttgart: Fraunhofer-Institut für Arbeitswirtschaft und Organisation IAO, 2017, S. 395-404

[4] Matthiesen, Sven ; Germann, René: Meaningful Prediction Parameters for Evaluating the Suitability of Power Tools for Usage. In: Procedia CIRP 70 (2018), S. 241-246

[5] Schenk, K. D. ; Vitalari, Nicholas P. ; Davis, K. Shannon: Differences between Novice and Expert Systems Analysts : What Do We Know and What Do We Do? In: Journal of Management Information Systems 15 (1998), Nr. 1, S. 9-50

[6] Bogner, Alexander ; Littig, Beate ; Menz, Wolfgang: Interviews mit Experten : Eine praxisorientierte Einführung. Wiesbaden : Springer VS, 2014 (Lehrbuch)

[7] Hitzler, Ronald ; Honer, Anne ; Maeder, Christoph: Expertenwissen : Die institutionalisierte Kompetenz zur Konstruktion von Wirklichkeit. Wiesbaden : Vieweg+Teubner Verlag, 1994

[8] Cellier, Jean-Marie ; Eyrolle, Hélène ; Marin, Claudette: Expertise in dynamic environments. In: Ergonomics 40 (1997), Nr. 1, S. 28-50

[9] Hreljac, Alan: Stride smoothness evaluation of runners and other athletes. In: Gait \& Posture 11 (2000), Nr. 3, S. 199-206

[10] Ganzevles, Sander P. M. ; Beek, Peter J. ; Daanen, Hein A. M. ; Coolen, Bart M. A. ; Truijens, Martin J.: Differences in swimming smoothness between elite and non-elite swimmers. In: Sports biomechanics (2019), S. 1-14 - Überprüfungsdatum 2020-01-31

[11] Anderson, Ross ; Breen, Sarah ; Tucker, Catherine: Movement Variability: A Comparison between Novic, Experienced and Elite Performers. In: ISBS Conference 2008, 2008

[12] Paulich, Monique ; Schepers, Martin ; Rudigkeit, Nina ; Bellusci, Giovanni: Xsens MTw Awinda: Miniature WirelessInertial-Magnetic Motion Tracker for HighlyAccurate 3D Kinematic Applications

[13] Cao, Zhe ; Hidalgo, Gines ; Simon, Tomas ; Wei, Shih-En ; Sheikh, Yaser: OpenPose: Realtime Multi-Person 2D Pose Estimation using Part Affinity Fields. 2018

This paper was corrected by the authors on 2020-12-15. In the original paper, the data presented in the paper for the joint angle of the right wrist was incorrectly referred to as position data of the right wrist. However, the conclusion drawn from the data regarding the variability of movement of the hand-arm system as an evaluation variable for the performance and expertice of the subjects remains valid. 